\title{
Redefining the prognostic likelihood of chronic lymphocytic leukaemia patients with borderline percentage of immunoglobulin variable heavy chain region mutations
}

\author{
Sara Raponi, ${ }^{1, \dagger}$ (ID Caterina Ilari, ${ }^{1, *}$ \\ Irene Della Starza, ${ }^{2}$ iD Luca V. \\ Cappelli, ${ }^{1}$ (iD Luciana Cafforio, ${ }^{1}$ \\ Alfonso Piciocchi, ${ }^{2}$ Valentina Arena, ${ }^{2}$ \\ Paola Mariglia, ${ }^{1}$ Francesca R. \\ Mauro, ${ }^{1}$ (D) Massimo Gentile, ${ }^{3,4}$ \\ Giovanna Cutrona, ${ }^{5}$ iD Riccardo \\ Moia, ${ }^{6}$ iD Chiara Favini, ${ }^{6}$ Fortunato \\ Morabito, ${ }^{4,7}$ Davide Rossi, ${ }^{8}$ Gianluca \\ Gaidano, ${ }^{6}$ Anna Guarini, ${ }^{9}$ Ilaria Del \\ Giudice $^{1, \dagger}$ (iD) and Robin Foà ${ }^{1, *}$ \\ ${ }^{1}$ Haematology, Department of \\ Translational and Precision Medicine, \\ Sapienza University of Rome, ${ }^{2}$ GIMEMA \\ Foundation, ${ }^{3}$ Haematology Unit, \\ Department of Haemato-Oncology, \\ Ospedale Annunziata, ${ }^{4}$ Aprigliano, \\ Biotechnology Research Unit, Cosenza, \\ ${ }^{5}$ Molecular Pathology Unit, IRCCS \\ Ospedale Policlinico San Martino, Genova, \\ ${ }^{6}$ Haematology, Department of \\ Translational Medicine, Amedeo Avogadro \\ University of Eastern Piedmont, Novara, \\ ${ }^{7}$ Haematology Department and Bone \\ Marrow Transplant Unit, Cancer Care \\ Center, Augusta Victoria Hospital, East \\ Jerusalem, Israel, ${ }^{8}$ Department of \\ Haematology, Oncology Institute of \\ Southern Switzerland and Institute of \\ Oncology Research, Bellinzona, Switzerland \\ and ${ }^{9}$ Department of Molecular Medicine, \\ Sapienza University, Rome, Italy
}

Received 2 September 2019; accepted for publication 19 November 2019

Correspondence: Prof. Robin Foà and Dr Ilaria Del Giudice, Haematology, Department of

Translational and Precision Medicine,

"Sapienza" University of Rome, Rome, Italy. E-mails: rfoa@bce.uniroma1.it; ilaria.delgiudice@uniromal.it

*These authors contributed equally to this study

\begin{abstract}
Summary
In chronic lymphocytic leukaemia (CLL), caution is warranted regarding the clinical implications of immunoglobulin variable heavy chain region (IGHV) rearrangements with a 'borderline' (BL) percentage of mutations (i.e. 97$97.9 \%$ IGHV identity). We analysed the IGHV mutational status in 759 untreated CLL patients (cohort 1). BL-CLL $(n=36,5 \%)$ showed a time to first treatment (TFT) similar to that of M-CLL $(n=338)$ and significantly longer than that of UM-CLL $(n=385)$, despite the enrichment in subset \#2 cases. In fact, CLLs belonging to subset \#2 $(n=15 / 759,2 \%)$ were significantly more frequent among BL-CLLs $(n=5 / 36,14 \%)$, with a brief TFT. TFT of BL-CLL remained comparable to that of M-CLL also considering the 327 CLL patients evaluated at diagnosis. These findings were then validated in an independent cohort 2 of 759 newly diagnosed CLL patients (BL-CLL: $n=11$, $1 \cdot 4 \%)$ and in all newly diagnosed patients from cohorts 1 and $2(n=1086$, $84 \%$ stage A; BL-CLL: $n=47,4 \cdot 3 \%)$. BL-CLL at diagnosis showed a biological profile comparable to that of M-CLL with a low frequency of unfavourable prognostic markers, except for a significant enrichment in subset \#2. Our data suggest that the prognosis of BL-CLL is good and similar to that of M-CLL, with the exception of subset $\# 2$ cases.
\end{abstract}

Keywords: chronic lymphocytic leukaemia, immunoglobulin variable heavy chain, somatic hypermutation, prognosis. 
The somatic hypermutation (SHM) of immunoglobulin heavy chain variable $(I G H V)$ region genes remains one of the strongest independent prognostic markers in chronic lymphocytic leukaemia (CLL) patients (Damle et al., 1999; Hamblin et al., 1999; Baliakas et al., 2019; Del Giudice \& Foà, 2019). The $98 \%$ cut-off of identity to the germline counterpart is recommended by the European Research Initiative on CLL (ERIC) guidelines (Rosenquist et al., 2017) and generally adopted in the current clinical practice to classify CLL patients into two subgroups with a different clinical course: the 'unmutated' CLL (UM-CLL, $\geq 98 \%$ ), characterized by an adverse prognosis, and the 'mutated' CLL (M-CLL, <98\%), that show a prolonged overall survival (OS) (Kröber et al., 2002; Lin et al., 2002). More recently, the IGHV status has acquired further relevance, being a valuable predictive marker of treatment efficacy besides TP53 deletion/mutation. At present, UM-CLL are candidates for B-cell receptor (BCR) inhibitors as first-line treatment, given the short progression-free survival (PFS) after chemoimmunotherapy, whilst M-CLL devoid of adverse cytogenetics can still benefit from first-line chemoimmunotherapy, usually followed by a prolonged PFS (Rossi et al., 2015).

CLL cases with a percentage of IGHV identity close to the $98 \%$ cut-off are defined as 'borderline' (BL). Their identification remains controversial as well as their clinical outcome. Davis et al. (2016) defined BL-CLL as those with IGHV identity between $97 \%$ and $98.99 \%$ and an intermediate outcome compared to UM- and M-CLL. The last ERIC guidelines identify BL-CLL as those whose IGHV identity is between 97\% and 97.99\%; since their clinical outcome remains undefined, caution is warranted in reporting the prognostic implications of this specific IGHV status to the clinicians and such a statement should be included in the laboratory report (Rosenquist et al., 2017). Beside the IGHV mutational status, the 'stereotyped' BCR can impact prognosis. In particular subset \#2, which carries the IGHV3-21 gene, is associated with an unfavourable prognosis, irrespective of the SHM status (Tobin et al., 2003), and it typically shows an IGHV identity close to $98 \%$ (Baliakas et al., 2015).

With the aim of investigating the prognostic significance of BL-CLL, we retrospectively analysed 759 untreated CLL patients (cohort 1) collected at our Institution, including 327 patients at diagnosis. Two independent series $(n=308$, $n=451$; cohort 2 ) of newly diagnosed CLL patients were used to validate the data.

\section{Patients and Methods}

\section{Patients}

We analysed IGHV gene rearrangements of 759 untreated CLL patients (cohort 1), collected at our Institution between 1988 and 2017. Of these, 327 (43\%) were newly diagnosed CLL (Binet stage A: 200 cases, 80\%), whilst 411 (54\%) were enrolled in different first-line GIMEMA (Gruppo Italiano Malattie EMatologiche dell'Adulto) trials. Two independent series ( $n=308, n=451$; cohort 2 ) of newly diagnosed CLL patients (Binet stage A: 709 cases, 93\%) were used to validate the data. The first series of cohort 2 is represented by Binet stage A CLL enrolled in a prospective trial (O-CLL1-GISL protocol, clinicaltrial.gov ID: NCT00917549) (Gentile et al., 2014); the second is represented by a monocentric prospective registry of newly diagnosed CLL (from 1985 to 2018).

\section{Analysis of IGHV-IGHD-IGHJ gene rearrangements}

$I G H V$ gene rearrangements were amplified using familyspecific VH primers [framework region 1 (FR1) or VH leader primers], combined with consensus $\mathrm{JH}$ primers and sequenced using the 3500 Series Genetic Analyzer (Thermo Fisher Scientific, Waltham, MA, USA) (Ghia et al., 2007). Following the ERIC guidelines that recommended the use of leader primers (Rosenquist et al., 2017), we resequenced old FR1 cases to avoid underestimating the IGHV SHM rate. All $I G H V$ gene rearrangements were re-aligned to the updated IMGT/V-QUEST tool (version 3.3.0; http://www.imgt.org/ IMGT_vquest/input) to determine a more accurate SHM status (Xochelli et al., 2015). Cases with 97-97.9\% IGHV identity were defined as BL-CLL (Rosenquist et al., 2017). To determine whether a particular IGHV gene rearrangement belongs to a major stereotyped subset, we used the ARResT/ AssignSubsets bioinformatics tool (bat.infspire.org/arrest/ericll.org/pages/services/tool) (Bystry et al., 2015).

\section{Statistical analysis}

Non-parametric tests were applied, in a univariate analysis, for comparisons between groups (chi-squared and Fisher exact test for difference of categorical variables or response rate, Mann-Whitney and Kruskal-Wallis test for difference of continuous variables).

Time to first treatment (TFT), calculated from the date of diagnosis to the date of first therapy or last follow-up, was estimated using the Kaplan-Meier Product Limit estimator. Subgroup comparisons were performed for descriptive purposes. Differences in terms of TFT were evaluated by means of a log-rank test in univariate analysis and by means of the Cox regression model in multivariate analysis, after assessment of the proportionality of hazards.

All the analyses were performed using the SAS system software (version 9.4; SAS Inc., Cary, NC, USA) or R software ( $\mathrm{R}$ Core Team, 2017). All tests were two-sided and confidence intervals were calculated at the $95 \%$ level.

\section{Results and Discussion}

Although the $98 \%$ cut-off of $I G H V$ identity to the germline counterpart is the approved value to clinically discriminate $\mathrm{UM}$ from M-CLL, for cases in which the percentage of 
identity is close to the cut-off (i.e. BL-CLL) the prognostic stratification still remains controversial (Davis et al., 2016; Rosenquist et al., 2017). In order to evaluate the prognosis of BL-CLL cases, we retrospectively analysed 759 untreated CLL patients. A total of $774 I G H V-D-J$ ( $I G H$ variable, diversity, joining) productive rearrangements were identified, with a single rearrangement in 744 cases and a double rearrangement with concordant SHM status in 15 cases; $382(50 \%)$ UM-CLL, 35 (5\%) BL-CLL and 342 (45\%) M-CLL were obtained. Given the historical collection of cases from cohort 1, we updated the $I G H V$ analysis in order to accurately determine the IGHV SHM rate (Rosenquist et al., 2017). First, among the 80 old cases sequenced with FR1 primers and showing a percentage of IGHV identity between 99.9 and $90 \cdot 1 \%, 62$ cases with available material were reanalysed with the leader primers. Six $(9 \cdot 7 \%)$ were reclassified as follows: three M-CLL $(96 \cdot 8 \%, 96 \cdot 7 \%, 96 \cdot 7 \%$, respectively) as BL-CLL (97.6\%, 97.9\%, 97.9\%, respectively), two BL-CLL (97.9\%, 97.8\%, respectively) as UM-CLL (98.7\%, 98.6\%, respectively) and one M-CLL (96.9\%) as UM-CLL (98\%). Second, all cases were re-aligned to the updated IMGT/VQUEST tool and none shifted across the three categories (UM/BL/M). Overall, 385 (51\%) UM-CLL, 36 (5\%) BL-CLL (mean \% of identity: 97.4\%) and 338 (44\%) M-CLL were identified, with a higher frequency of UM-CLL due to the enrichment of cases enrolled in first-line clinical trials. Cluster analysis of the heavy complementarity-determining region 3 (HCDR3) allowed us to identify 15/759 (2\%) subset \#2 cases: six cases in UM-CLL (1\%), five in BL-CLL (14\%) and four in M-CLL (1\%). Thus, we confirmed a significantly higher frequency of this subset in the $\mathrm{BL}$ category with respect to UM- and M-CLL $(P<0.0001)$, in agreement with the reported enrichment of subset $\# 2$ among cases with a low mutational load (Baliakas et al., 2015).

The clinical impact of the BL percentage of $I G H V$ mutations is not completely defined. Cases with an IGHV identity between $97 \%$ and $98.99 \%$ were defined by Davis et al. (2016) as an intermediate prognostic group compared to UM- and M-CLL in terms of TFT, PFS and OS. However, the recent ERIC guidelines define as BL-CLL cases those with an IGHV identity between $97 \%$ and $97.99 \%$ and underline their uncertain clinical outcome (Rosenquist et al., 2017).

In our series, UM-CLL cases showed a shorter TFT than M-CLL $(P<0.0001)$ (median follow-up: 120 months; range 0-120), according to the literature (Damle et al., 1999; Hamblin et al., 1999) (data not shown). We could confirm the

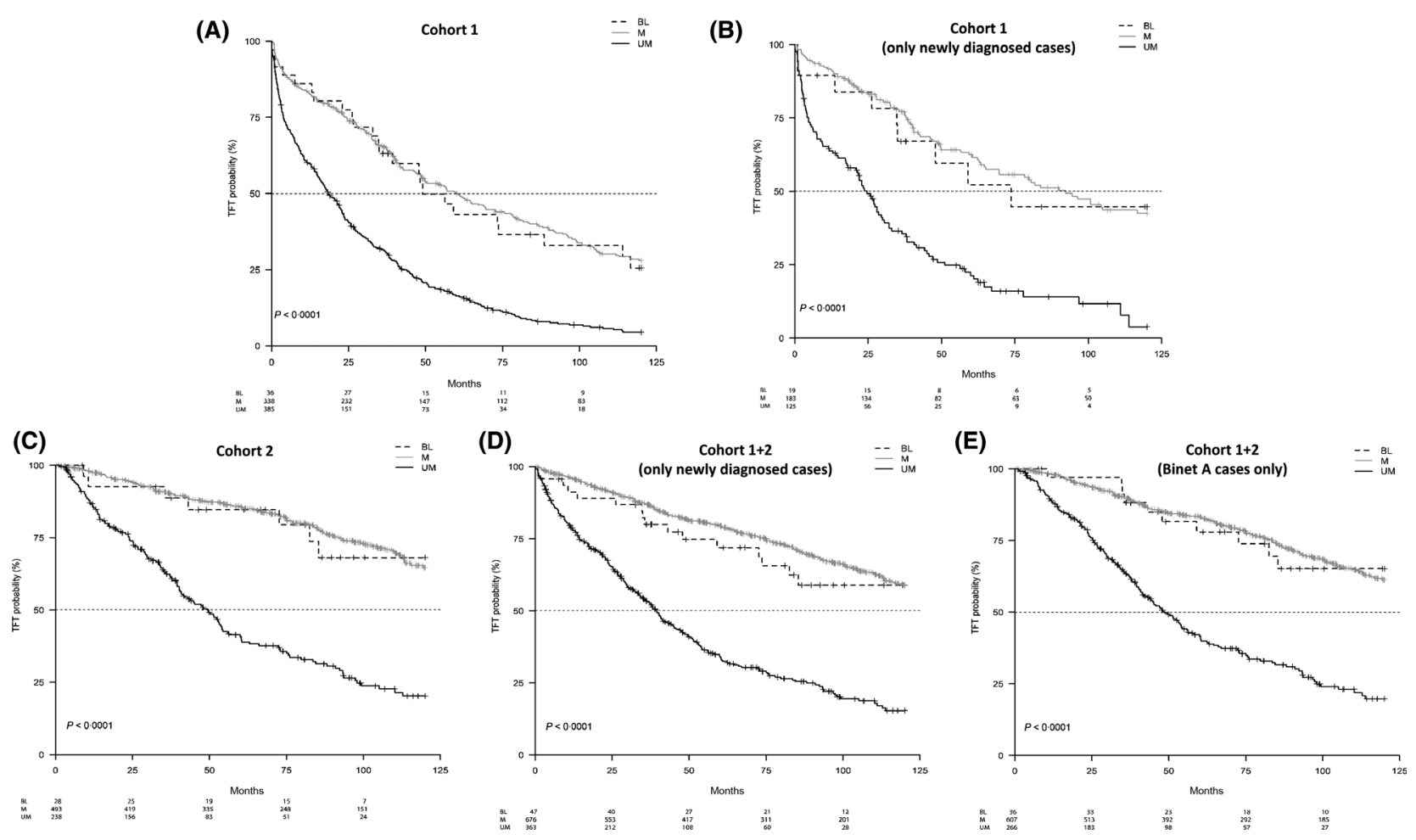

Fig 1. Time to first treatment (TFT) analysis. (A) TFT of cohort $1(n=759)$ : UM $(n=385)$ versus BL $(n=36)$ versus M-CLL (chronic lymphocytic leukaemia) $(n=338)$. $P$ value: $\mathrm{BL}$ versus $\mathrm{M}=$ n.s.; BL versus $\mathrm{UM}<0.0001 ; \mathrm{M}$ versus $\mathrm{UM}<0.0001$; $(\mathrm{B})$ TFT of newly diagnosed CLL patients from cohort $1(n=327)$ : $\mathrm{UM}(n=125)$ versus $\mathrm{BL}(n=19)$ versus $\mathrm{M}-\mathrm{CLL}(n=183)$. $P$ value: $\mathrm{BL}$ versus $\mathrm{M}=\mathrm{n} . \mathrm{s} . ; \mathrm{BL}$ versus $\mathrm{UM}<0.0001 ; \mathrm{M}$ versus $\mathrm{UM}<0.0001$; (C) TFT of validation cohort $2(n=759)$ : $\mathrm{UM}(n=238)$ versus BL $(n=28)$ versus M-CLL $(n=493) . P$ value: $\mathrm{BL}$ versus $\mathrm{M}=$ n.s.; $\mathrm{BL}$ versus $\mathrm{UM}<0 \cdot 0001$; (D) TFT of newly diagnosed CLL from cohort 1 and validation cohort 2 ( $n=1086)$ : $\mathrm{UM}$ $(n=363)$ versus $\mathrm{BL}(n=47)$ versus $\mathrm{M}-\mathrm{CLL}(n=676)$. $P$ value: $\mathrm{BL}$ versus $\mathrm{M}=\mathrm{n} . \mathrm{s}$; $\mathrm{BL}$ versus $\mathrm{UM}<0 \cdot 0001 ;$ (E). TFT of Binet stage A CLL patients $(n=909)$ : UM $(n=266)$ versus $\mathrm{BL}(n=36)$ versus $\mathrm{M}-\mathrm{CLL}(n=607) . P$ value: $\mathrm{BL}$ versus $\mathrm{M}=\mathrm{n} . \mathrm{s} . ; \mathrm{BL}$ versus $\mathrm{UM}<0 \cdot 0001$. $\mathrm{UM}$, unmutated; BL, borderline; M, mutated; CLL, chronic lymphocytic leukaemia; n.s., not significant. 
Table I. Biological features of newly diagnosed patients from cohorts 1 and $2(n=1086)$

\begin{tabular}{|c|c|c|c|c|c|c|}
\hline & $\mathrm{UM}-I G H V(n=363)$ & BL-IGHV $(n=47)$ & M-IGHV $(n=676)$ & $\begin{array}{l}\mathrm{M} \text { vs. } \mathrm{UM}-I G H V \\
P \text { value }\end{array}$ & $\begin{array}{l}\text { UM vs. } \\
\text { BL- } I G H V \\
P \text { value }\end{array}$ & $\begin{array}{l}\mathrm{M} v s . \\
\mathrm{BL}-I G H V \\
P \text { value }\end{array}$ \\
\hline Median age (range) & $61 \cdot 5(26-92)$ & $61(38-83)$ & $61(27-92)$ & 0.778 & 0.338 & $0 \cdot 265$ \\
\hline Gender (male/female) & $197 / 165$ & $32 / 15$ & $342 / 334$ & $0 \cdot 266$ & $0 \cdot 105$ & 0.030 \\
\hline $\begin{array}{l}\mathrm{CD} 8^{+} \\
\text {(cut-off: } 30 \% \text { ) }\end{array}$ & $87 / 197(44 \%)$ & $5 / 29(17 \%)$ & $25 / 342(7 \%)$ & $<0.0001$ & $0 \cdot 0076$ & 0.07 \\
\hline $\begin{array}{l}\text { ZAP70 } \\
\text { (cut-off: } 30 \% \text { ) }\end{array}$ & 136/191 (71\%) & $11 / 28(39 \%)$ & $70 / 327(21 \%)$ & $<0 \cdot 0001$ & 0.002 & 0.04 \\
\hline del11q/del17p & $83 / 326(25 \%)$ & $4 / 39(10 \%)$ & $26 / 625(4 \%)$ & $<0.0001$ & 0.044 & 0.09 \\
\hline TP53 mutation & 22 MUT/306 WT (7\%) & 3 MUT/34 WT (8\%) & 22 MUT/580 WT (4\%) & 0.05 & 0.73 & $0 \cdot 17$ \\
\hline TP53 disruption & $16 / 296(5 \%)$ & $2 / 33(6 \%)$ & $14 / 568(2 \cdot 5 \%)$ & 0.03 & 0.69 & $0 \cdot 22$ \\
\hline NOTCH1 mutation & 62 MUT/168 WT (27\%) & $1 \mathrm{MUT} / 22 \mathrm{WT}(4 \%)$ & 13 MUT/412 WT (3\%) & $<0.0001$ & 0.02 & $0 \cdot 53$ \\
\hline Subset \#2 & $6 / 363(2 \%)$ & $5 / 47(11 \%)$ & $4 / 675(0 \cdot 6 \%)$ & $0 \cdot 10$ & $<0 \cdot 0001$ & $<0 \cdot 0001$ \\
\hline
\end{tabular}

$\mathrm{UM}$, unmutated; BL, borderline; $\mathrm{M}$, mutated; $I G H V$, immunoglobulin variable heavy chain.

poor outcome of subset \#2 cases when compared to M-CLL $(P<0.0001)$ (Figure S1). BL-CLL cases showed a significantly longer TFT compared to UM-CLL $(P<0 \cdot 0001)$ and similar to that of M-CLL (Fig 1A). When we excluded all subset \#2 cases from the outcome analyses, the TFT of the BL-CLL remained unchanged. To avoid a bias of selection, half of our cases being enrolled in different first-line clinical trials, we separately analysed the 327 patients evaluated on diagnosis ( $80 \%$ stage Binet A), 125 of which were UM-CLL (38\%), 19 BL-CLL (6\%) and 183 M-CLL (56\%). Also in this subgroup, BL-CLL cases maintained a significantly longer TFT than UM-CLL $(P<0.0001)$ and similar to that of $\mathrm{M}$ CLL, as a whole (Fig 1B), also after the exclusion of subset \#2 cases $(P<0.0001)$ (data not shown). The same results were obtained in the remaining 432 patients evaluated at the time of progression and first-line treatment (data not shown).

All these findings were subsequently validated in cohort 2 (median follow-up: 90.2 months; range 0.27-120), which included two independent series of 308 and 451 newly diagnosed CLL patients. In this cohort, 238 (31\%) UM-CLL, 28 (4\%) BL-CLL, 493 (65\%) M-CLL were identified; 709 were Binet stage A CLL and subset \#2 was present in 11/758 ( $1.4 \%$ ) cases (the stereotypy of one case was not available). In addition, in this cohort the TFT of BL-CLL was similar to that of M-CLL (Fig 1C).

When all newly diagnosed patients from cohorts 1 and 2 were pooled together $(n=1086,84 \%$ Binet stage A; median follow-up: 90.5 months; range 0-120), 363 (34\%) UM-CLL, 47 (4\%) BL-CLL and 676 (62\%) M-CLL were identified. The TFT of BL-CLL remained similar to that of M-CLL (Fig 1D, S2) and the results were confirmed also in the 909 Binet stage A CLL (Fig 1E).

With regard to the cluster analysis of the HCDR3 region, available in 1085 cases, 69/1085 (6.3\%) showed a major stereotyped rearrangement with known prognostic value.
Subset \#1 recurred in 20/1085 cases (1.8\%), subset \#2 in 15/ 1085 (1.4\%), subset \#4 in 30/1085 (2.8\%) and subset \#8 in $5 / 1085(0 \cdot 5 \%)$. As expected, BL-CLL were enriched in subset $\# 2$ cases $(5 / 47,11 \%)$, and subset \#8 and \#1 cases belonged to UM-CLL, whilst all but one subset \#4 cases were M-CLL (Del Giudice et al., 2014; Baliakas et al., 2015; Stamatopoulos et al., 2017).

Looking at the biological features of the 1086 CLL patients at diagnosis, as expected (Kröber et al., 2002; Lin et al., 2002; Rassenti et al., 2008), UM-CLL were significantly different from M-CLL (Table I). With the exception of an enrichment of subset \#2, BL-CLL showed a biological profile not significantly different from M-CLL: CD $38^{+}: 17 \%$ vs. 7\%; del11q/ del17p ${ }^{+}: 10 \%$ vs. $4 \%$; NOTCH1-mutated: $4 \%$ vs. $3 \%$; TP53 mutation: $8 \%$ vs. $4 \%$, respectively. All these results held true also considering the 909 Binet stage A CLL patients only (data not shown).

In an univariate analysis including age, gender, CD38, FISH (unfavourable vs. favourable lesions), TP53 and NOTCH1 mutations, subset \#2 and the percentage of IGHV identity, the factors significantly associated with TFT were CD38, FISH, NOTCH1 mutations and the IGHV percentage of identity ( $<98 \%$, M-CLL vs $\geq 98 \%$, UM-CLL). The multivariate analysis identified CD38, FISH and IGHV percentages of identity (M-CLL versus UM-CLL) as independent prognostic markers for TFT. The BL percentage of IGHV identity per se did not reach statistical significance in the univariate nor in the multivariate analyses, confirming that its prognostic significance is not different from that of M-CLL (Table S1).

Finally, we divided patients according to the IGHV percentage of identity and tested the TFT in the following subgroups: 99-100\%, 98-98.99\%, 97-97.99\% (BL-CLL) and $<97 \%$. In all the analyses, performed on cohort 1 , on the newly diagnosed cases and on the Binet stage A patients, with or without subset \#2 cases, the subgroup with 
98-98.99\% IGHV identity showed a significantly better TFT than cases with $99-100 \%$ IGHV identity and superimposable with that of BL-CLL cases (Figure S3), in contrast with Davis et al. (2016). Recent papers have revisited the clinical significance of the IGHV mutation load in CLL, which remains somehow controversial. Jain et al. have reported that in FCR (fludarabine-cyclophosphamide-rituximab)-treated patients, the absolute level of $I G H V$ percentage of identity rather than the $98 \%$ cut-off is a better predictor of post-treatment outcome, pointing to the potential relevance of the SHM as a continuous rather than a dichotomized variable to predict FCR efficacy (Jain et al., 2018). A subsequent study on 1003 CLL patients, including the newly diagnosed Binet stage A patients from protocol O-CLL1, did not confirm the value of SHM as a continuous variable in the prognostic assessment of TFT (Morabito et al., 2018), reinforcing the 98\% cut-off as the best cut-off to distinguish two groups with a different prognostic likelihood, without the need for a further subdivision.

Genome-wide DNA methylation studies led to the concept of new clinical-biological CLL subgroups based on the putative leukaemic cell of origin and identified by different epigenetic signatures corresponding to different degrees of B-cell maturation. On these bases, three groups have been identified, namely naive (n-CLL), memory (m-CLL) and intermediate CLL (i-CLL): n-CLL mostly included cases with unmutated IGHV, m-CLL mostly mutated IGHV CLL with low levels of IGHV identity and i-CLL (11-32\% of all CLL) mostly mutated IGHV cases with intermediate mutational load, but also unmutated IGHV (Queiros et al.., 2015; Wojdacz et al., 2019). Thus, although our BL-CLL would likely fall within the i-CLL, there is no absolute correspondence between the percentage of IGHV identity and the epigenetic signature. i-CLL, enriched in SF3B1 mutations and subset \#2, showed an intermediate outcome in TFT and OS with respect to $\mathrm{n}$-CLL and $\mathrm{m}$-CLL, as well as an inferior outcome after chemo/chemoimmunotherapy compared to m-CLL (Queiros et al.., 2015; Wojdacz et al., 2019). The classification of CLL patients on the basis of the cell of origin, though not widely available, is intriguing from a biological point of view and, if validated, might be also relevant to identify those mutated IGHV cases that go less well than expected, with a potential clinical implication.

Our data strongly suggest that BL-CLL patients have the same prognosis of M-CLL, showing similar biological features with a low frequency of unfavourable prognostic markers. Notably, these results hold true in newly diagnosed and in Binet stage A CLL. Thus, CLL with IGHV identity below $98 \%$ can be considered a group with a good prognosis, with the exception of subset \#2 cases. Given the relevance of the IGHV mutational status to guide therapeutic decisions in CLL patients, besides TP53 disruption, an accurate determination of the SHM rate and BCR stereotypy analysis are mandatory, especially in cases with a low mutational load (Baliakas et al., 2018; Jaramillo et al.., 2019). The issue of the outcome of BL-CLL post treatment (i.e. after chemoimmunotherapy and after BCR inhibitors) remains open and of great relevance.

\section{Acknowledgements}

The authors wish to thank Associazione Italiana per la Ricerca sul Cancro (AIRC), Metastases Special Program, $\mathrm{N}^{\circ}$ 21198, Milan, Italy (RF); Ricerca finalizzata RF-201102349712 (RF); Finanziamento Medi Progetti Universitari 2014, C26A14WWA9 (IDG); Progetti di Rilevante Interesse Nazionale (PRIN) 2015ZMRFEA (RF).

\section{Author Ccontributions}

SR and CI designed the study, analysed the results and wrote the paper; IDS and IDG designed the study and critically revised the manuscript; LVC contributed to analysis of the results; CI, LC and GC performed and analysed Sanger sequencing; $\mathrm{AP}$ and VA performed the statistical analysis; PM provided samples; FRM, MG, RM, CF, DR, GG, FM managed patients and provided the clinico-biological data; AG discussed the results and critically revised the manuscript; RF contributed to the design of the study and critically revised the manuscript.

\section{Conflicts of interest}

The authors have no conflicts of interest to disclose.

\section{Supporting Information}

Additional supporting information may be found online in the Supporting Information section at the end of the article.

Figure S1. Time to first treatment (TFT) analysis in cohort $1(\mathrm{n}=759)$ : subset \#2 $(n=15)$ vs M-CLL $(n=365)$ vs UM-CLL $(n=379)$ ( $P$ value $<0.0001)$.

Figure S2. Time to first treatment (TFT) analysis in newly diagnosed CLL $(n=1085)$ : subset \#2 $(n=15)$ vs non-subset \#2 M-CLL $(n=587)$ vs. non-subset \#2 UM-CLL $(n=441)$ vs non-subset \#2 BL-CLL $(n=42)(P$ value $<0.0001)$.

Figure S3. Time to first treatment (TFT) analysis of patients subdivided according to the $I G H V$ percentage of identity as follows: $99-100 \%$ (1) vs. $98-98.99 \%$ (2) vs. $97-$ 97.99\% (3, BL-CLL) vs. $<97 \%$ (4). (S3a) cohort $1(n=759)$; (S3b) newly diagnosed CLL from cohort 1 and validation cohort $2 \quad(n=1086)$; S3c $)$ Binet stage A CLL patients $(n=909)$.

Table SI. Univariate and multivariate analysis in the newly diagnosed CLL from cohort 1 and validation cohort 2 $(n=1086)$. 


\section{References}

Baliakas, P., Agathangelidis, A., Hadzidimitriou, A., Sutton, L.A., Minga, E., Tsanousa, A., Scarfò, L., Davis, Z., Yan, X.J., Shanafelt, T., Plevova, K., Sandberg, Y., Vojdeman, F.J., Boudjogra, M., Tzenou, T., Chatzouli, M., Chu, C.C., Veronese, S., Gardiner, A., Mansouri, L., Smedby, K.E., Pedersen, L.B., Moreno, D., Van Lom, K., Giudicelli, V., Francova, H.S., Nguyen-Khac, F., Panagiotidis, P., Juliusson, G., Angelis, L., Anagnostopoulos, A., Lefranc, M.P., Facco, M., Trentin, L., Catherwood, M., Montillo, M., Geisler, C.H., Langerak, A.W., Pospisilova, S., Chiorazzi, N., Oscier, D., Jelinek, D.F., Darzentas, N., Belessi, C., Davi, F., Ghia, P., Rosenquist, R. \& Stamatopoulos, K. (2015) Not all IGHV3-21 chronic lymphocytic leukemias are equal: prognostic considerations. Blood, 125, 856-859.

Baliakas, P., Mattsson, M., Hadzidimitriou, A., Minga, E., Agathangelidis, A., Sutton, L.A., Scarfo, L., Davis, Z., Yan, X.J., Plevova, K., Sandberg, Y., Vojdeman, F.J., Tzenou, T., Chu, C.C., Veronese, S., Mansouri, L., Smedby, K.E., Giudicelli, V., Nguyen-Khac, F., Panagiotidis, P., Juliusson, G., Anagnostopoulos, A., Lefranc, M.P., Trentin, L., Catherwood, M., Montillo, M., Niemann, C.U., Langerak, A.W., Pospisilova, S., Stavroyianni, N., Chiorazzi, N., Oscier, D., Jelinek, D.F., Shanafelt, T., Darzentas, N., Belessi, C., Davi, F., Ghia, P., Rosenquist, R. \& Stamatopoulos, K. (2018) No improvement in long-term survival over time for chronic lymphocytic leukemia patients in stereotyped subsets \#1 and \#2 treated with chemo(immuno) therapy. Haematologica, 103, e158-e161.

Baliakas, P., Moysiadis, T., Hadzidimitriou, A., Xochelli, A., Jeromin, S., Agathangelidis, A., Mattsson, M., Sutton, L.A., Minga, E., Scarfò, L., Rossi, D., Davis, Z., Villamor, N., Parker, H., Kotaskova, J., Stalika, E., Plevova, K., Mansouri, L., Cortese, D., Navarro, A., Delgado, J., Larrayoz, M., Young, E., Anagnostopoulos, A., Smedby, K.E., Juliusson, G., Sheehy, O., Catherwood, M., Strefford, J.C., Stavroyianni, N., Belessi, C., Pospisilova, S., Oscier, D., Gaidano, G., Campo, E., Haferlach, C., Ghia, P., Rosenquist, R. \& Stamatopoulos, K. (2019) Tailored approaches grounded on immunogenetic features for refined prognostication in chronic lymphocytic leukemia. Haematologica, 104, 360-369.

Bystry, V., Agathangelidis, A., Bikos, V., Sutton, L.A., Baliakas, P., Hadzidimitriou, A., Stamatopoulos, K. \& Darzentas, N.; European Research Initiative on CLL (2015) ARResT/ AssignSubsets: a novel application for robust subclassification of chronic lymphocytic leukemia based on B cell receptor IG stereotypy. Bioinformatics, 31, 3844-3846.

Damle, R.N., Wasil, T., Fais, F., Ghiotto, F., Valetto, A., Allen, S.L., Buchbinder, A., Budman, D., Dittmar, K., Kolitz, J., Lichtman, S.M., Schulman, P., Vinciguerra, V.P., Rai, K.R., Ferrarini, M. \& Chiorazzi, N. (1999) Ig V gene mutation status and CD38 expression as novel prognostic indicators in chronic lymphocytic leukemia. Blood, 94, 1840-1847.

Davis, Z., Forconi, F., Parker, A., Gardiner, A., Thomas, P., Catovsky, D., Rose-Zerilli, M., Strefford, J.C. \& Oscier, D. (2016) The outcome of chronic lymphocytic leukaemia patients with 97\% IGHV gene identity to germline is distinct from cases with $<97 \%$ identity and similar to those with $98 \%$ identity. British Journal of Haematology, 173, 127-136.

Del Giudice, I. \& Foà, R. (2019) Another step forward in the 20-year history of IGHV mutations in chronic lymphocytic leukemia. Haematologica, 104, 219-221.

Del Giudice, I., Chiaretti, S., Santangelo, S., Tavolaro, S., Peragine, N., Marinelli, M., Ilari, C., Raponi, S., Messina, M., Nanni, M., Mauro, F.R., Piciocchi, A., Bontempi, K., Rossi, D., Gaidano, G., Guarini, A. \& Foà, R. (2014) Stereotyped subset \#1 chronic lymphocytic leukemia: a direct link between B-cell receptor structure, function, and patients' prognosis. American Journal of Hematology, 89, 74-82.

Gentile, M., Cutrona, G., Mosca, L., Matis, S., Fabris, S., Lionetti, M., Ilariucci, F., Zupo, S., Musolino, C., Levato, L., Molica, S., Di Raimondo, F., Vincelli, I., Di Rienzo, N., Pesce, E.A., Angrilli, F., Federico, M., Neri, A., Ferrarini, M. \& Morabito, F. (2014) Prospective validation of a risk score based on biological markers for predicting progression free survival in Binet stage A chronic lymphocytic leukemia patients: results of the multicenter O-CLL1-GISL study. American Journal of Hematology, 89, 743750.

Ghia, P., Stamatopoulos, K., Belessi, C., Moreno, C., Stilgenbauer, S., Stevenson, F., Davi, F. \& Rosenquist, R. (2007) ERIC recommendations on IGHV gene mutational status analysis in chronic lymphocytic leukemia. Leukemia, 21, 13.

Hamblin, T.J., Davis, Z., Gardiner, A., Oscier, D.G. \& Stevenson, F.K. (1999) Unmutated Ig V $(\mathrm{H})$ genes are associated with a more aggressive form of chronic lymphocytic leukemia. Blood, 94, 1848-1854.

Jain, P., Nogueras González, G.M., Kanagal-Shamanna, R., Rozovski, U., Sarwari, N., Tam, C., Wierda, W.G., Thompson, P.A., Jain, N., Luthra, R., Quesada, A., Sanchez-Petitto, G., Ferrajoli, A., Burger, J., Kantarjian, H., Cortes, J., O’Brien, S., Keating, M.J. \& Estrov, Z. (2018) The absolute percent deviation of IGHV mutation rather than a $98 \%$ cut-off predicts survival of chronic lymphocytic leukaemia patients treated with fludarabine, cyclophosphamide and rituximab. British Journal of Haematology, 180, 33-40.

Jaramillo Segura, S., Agathangelidis, A., Schneider, C., Bahlo, J., Robrecht, S., Tausch, E., Bloehdorn, J., Hoechstetter, M., Fischer, K., Eichhorst, B., Goede, V., Hallek, M., Döhner, H., Rosenquist, R., Ghia, P., Stamatopoulos, K. \& Stilgenbauer, S. (2019). Prognostic impact of common chronic lymphocytic leukemia stereotyped subsets: analysis within prospective clinical trials of the German CLL Study Group (GCLLSG). Abs n. 2051. International Workshop on CLL, 20-23 September 2019, Edinburgh.

Kröber, A., Seiler, T., Benner, A., Bullinger, L., Brückle, E., Lichter, P., Döhner, H. \& Stilgenbauer, S. (2002) V(H) mutation status, CD38 expression level, genomic aberrations, and survival in chronic lymphocytic leukemia. Blood, 100, 1410-1416.

Lin, K., Sherrington, P.D., Dennis, M., Matrai, Z., Cawley, J.C. \& Pettitt, A.R. (2002) Relationship between p53 dysfunction, CD38 expression, and $\operatorname{IgV}(\mathrm{H})$ mutation in chronic lymphocytic leukemia. Blood, 100, 1404-1409.

Morabito, F., Shanafelt, T.D., Gentile, M., Reda, G., Mauro, F.R., Rossi, D., Di Renzo, N., Molica, S., Angrilli, F., Chiarenza, A., Cutrona, G., Chaffee, K.G., Parikh, S.A., Tripepi, G., D’Arrigo, G., Vigna, E., Recchia, A.G., Cortelezzi, A., Gaidano, G., Di Raimondo, F., Fais, F., Foà, R., Neri, A. \& Ferrarini, M. (2018) Immunoglobulin heavy chain variable region gene and prediction of time to first treatment in patients with chronic lymphocytic leukemia: Mutational load or mutational status? Analysis of 1003 cases. American Journal of Hematology, 93, E216-E219.

Queirós, A.C., Villamor, N., Clot, G., MartinezTrillos, A., Kulis, M., Navarro, A., Penas, E.M., Jayne, S., Majid, A., Richter, J., Bergmann, A.K., Kolarova, J., Royo, C., Russiñol, N., Castellano, G., Pinyol, M., Bea, S., Salaverria, I., LópezGuerra, M., Colomer, D., Aymerich, M., Rozman, M., Delgado, J., Giné, E., González-Díaz, M., Puente, X.S., Siebert, R., Dyer, M.J., LópezOtín, C., Rozman, C., Campo, E., López-Guillermo, A. \& Martín-Subero, J.I. (2015) A B-cell epigenetic signature defines three biologic subgroups of chronic lymphocytic leukemia with clinical impact. Leukemia, 29, 598-605.

Rassenti, L.Z., Jain, S., Keating, M.J., Wierda, W.G., Grever, M.R., Byrd, J.C., Kay, N.E., Brown, J.R., Gribben, J.G., Neuberg, D.S., He, F., Greaves, A.W., Rai, K.R. \& Kipps, T.J. (2008) Relative value of ZAP-70, CD38, and immunoglobulin mutation status in predicting aggressive disease in chronic lymphocytic leukemia. Blood, 112, 1923-1930.

Rosenquist, R., Ghia, P., Hadzidimitriou, A., Sutton, L.A., Agathangelidis, A., Baliakas, P., Darzentas, N., Giudicelli, V., Lefranc, M.P., Langerak, A.W., Belessi, C., Davi, F. \& Stamatopoulos, K. (2017) Immunoglobulin gene sequence analysis in chronic lymphocytic leukemia: updated ERIC recommendations. Leukemia, 31, 1477-1481.

Rossi, D., Terzi-di-Bergamo, L., De Paoli, L., Cerri, M., Ghilardi, G., Chiarenza, A., Bulian, P., Visco, C., Mauro, F.R., Morabito, F., Cortelezzi, A., Zaja, F., Forconi, F., Laurenti, L., Del Giudice, I., Gentile, M., Vincelli, I., Motta, M., 
Coscia, M., Rigolin, G.M., Tedeschi, A., Neri, A., Marasca, R., Perbellini, O., Moreno, C., Del Poeta, G., Massaia, M., Zinzani, P.L., Montillo, M., Cuneo, A., Gattei, V., Foà, R. \& Gaidano, G. (2015) Molecular prediction of durable remission after first-line fludarabine-cyclophosphamide-rituximab in chronic lymphocytic leukemia. Blood, 126, 1921-1924.

Stamatopoulos, K., Agathangelidis, A., Rosenquist, R. \& Ghia, P. (2017) Antigen receptor stereotypy in chronic lymphocytic leukemia. Leukemia, 31, 282-291.

Tobin, G., Thunberg, U., Johnson, A., Eriksson, I., Soderberg, O., Karlsson, K., Merup, M., Juliusson, G., Vilpo, J., Enblad, G., Sundstrom, C., Roos, G. \& Rosenquist, R. (2003) Chronic lymphocytic leukemias utilizing the VH3-21 gene display highly restricted Vlambda2-14 gene use and homologous CDR3s: implicating recognition of a common antigen epitope. Blood, 101, 4952-4957.

Wojdacz, T.K., Amarasinghe, H.E., Kadalayil, L., Beattie, A., Forster, J., Blakemore, S.J., Parker, H., Bryant, D., Larrayoz, M., Clifford, R., Robbe, P., Davis, Z.A., Else, M., Howard, D.R., Stamatopoulos, B., Steele, A.J., Rosenquist, R., Collins, A., Pettitt, A.R., Hillmen, P., Plass, C., Schuh, A., Catovsky, D., Oscier, D.G., Rose-Zerilli, M.J.J., Oakes, C.C. \& Strefford, J.C. (2019) Clinical significance of DNA methylation in chronic lymphocytic leukemia patients: results from 3 UK clinical trials. Blood Adv, 3, 2474-2481.
Xochelli, A., Agathangelidis, A., Kavakiotis, I., Minga, E., Sutton, L.A., Baliakas, P., Chouvarda, I., Giudicelli, V., Vlahavas, I., Maglaveras, N., Bonello, L., Trentin, L., Tedeschi, A., Panagiotidis, P., Geisler, C., Langerak, A.W., Pospisilova, S., Jelinek, D.F., Oscier, D., Chiorazzi, N. Darzentas, N., Davi, F., Ghia, P., Rosenquist, R., Hadzidimitriou, A., Belessi, C., Lefranc, M.P. \& Stamatopoulos, K. (2015) Immunoglobulin heavy variable (IGHV) genes and alleles: new entities, new names and implications for research and prognostication in chronic lymphocytic leukaemia. Immunogenetics, $\mathbf{6 7}$, 61-66. 